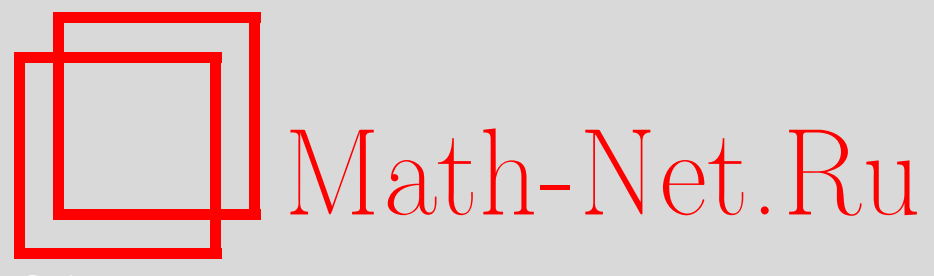

В. Н. Желябин, Корадикал йордановой (альтернативной) коалгебры и квазирегулярный радикал ее дуальной алгебры, Матем. заметки, 2006, том 80, выпуск 4, 509 515

DOI: https://doi.org/10.4213/mzm2843

Использование Общероссийского математического портала Math-Net.Ru подразумевает, что вы прочитали и согласны с пользовательским соглашением http://www . mathnet.ru/rus/agreement

Параметры загрузки:

IP : 54.84 .234 .179

26 апреля 2023 г., 18:28:08

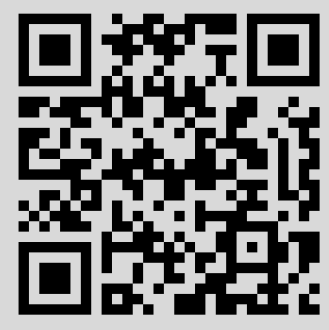




\section{КОРАДИКАЛ ЙОРДАНОВОЙ (АЛЬТЕРНАТИВНОЙ) КОАЛГЕБРЫ И КВАЗИРЕГУЛЯРНЫЙ РАДИКАЛ ЕЕ ДУАЛЬНОЙ АЛГЕБРЫ}

\section{В. Н. Желябин}

Доказано, что ортогональное дополнение корадикала йордановой (альтернативной) коалгебры совпадает с квазирегулярным радикалом ее дуальной алгебры.

Библиография: 5 названий.

Понятием, дуальным к понятию алгебры над полем, является понятие коалгебры. Одним из основных понятий в теории ассоциативных коалгебр и алгебр Хопфа является понятие корадикала. В [1] доказано, что ортогональное дополнение корадикала ассоциативной коалгебры совпадает с радикалом Джекобсона ее дуальной алгебры. Отсюда, в частности, следует, что радикал Джекобсона (квазирегулярный радикал) дуальной алгебры ассоциативной коалгебры замкнут в конечной (*-слабой) топологии.

Альтернативные и йордановы коалгебры были определены в работе [2].

В данной работе доказано, что квазирегулярный радикал дуальной алгебры йордановой (альтернативной) коалгебры замкнут в конечной топологии.

1. Определения и предварительные результаты. Пусть $V$ - линейное пространство над полем $\Phi$. Через $V^{*}$ обозначим дуальное пространство для $V$. Пусть $U$ - подмножество линейного пространства $V$. Тогда положим

$$
U^{\perp}=\left\{f \in V^{*} \mid f(u)=0 \text { для любого элемента } u \in U\right\} .
$$

Аналогично определяется $U^{\perp}$ для подмножества $U$ из $V^{*}$.

Дуальное пространство $V^{*}$ - линейное топологическое пространство. Базис окрестностей элемента $f$ из $V^{*}$ этой топологии образуют множества вида $f+W^{\perp}$, где $W$ - произвольное конечномерное подпространство из $V$. Данная топология называется конечной топологией. Замкнутыми в конечной топологии подпространствами являются подпространства вида $U^{\perp \perp}$, где $U$ - подпространство из $V^{*}$.

Для линейных пространств $V$ и $U$ над полем $\Phi$ через $V \otimes U$ обозначим их тензорное произведение над $\Phi$. Как известно, отображение $\rho: V^{*} \otimes V^{*} \mapsto(V \otimes V)^{*}$, определенное следующим правилом:

$$
\rho(f \otimes g)\left(\sum_{i} v_{i} \otimes u_{i}\right)=\sum_{i} f\left(v_{i}\right) g\left(u_{i}\right)
$$

Работа выполнена при поддержке фонда FAPESP, грант № 04/08537-4, и Министерства образования РНПВШ, грант № 11617.

(C) В.Н. ЖЕлявин, 2006 
является вложением. Если $\phi: V \mapsto U$ - линейное отображение линейных пространств, то сопряженное к нему линейное отображение $\phi^{*}: U^{*} \mapsto V^{*}$ задается правилом: $\left(\phi^{*}\left(u^{*}\right)\right)(v)=u^{*}(\phi(v))$, здесь $v \in V, u^{*} \in U^{*}$.

ОПределениЕ. Пара $(A, \Delta)$, где $A$ - линейное пространство, а $\Delta: A \mapsto A \otimes A-$ линейное отображение, называется коалгеброй. Отображение $\Delta$ называется коумножением. Для элемента $а$ коалгебры $(A, \Delta)$, если $\Delta(a)=\sum_{i} a_{1 i} \otimes a_{2 i}$, то, следуя Свидлеру [3], будем писать $\Delta(a)=\sum_{a} a_{(1)} \otimes a_{(2)}$.

Коединицей коалгебры $(A, \Delta)$ называется такой функционал $\epsilon$ из $A^{*}$, что для произвольного элемента $a$ из $A$ имеем

$$
a=\sum_{a} \epsilon\left(a_{(1)}\right) a_{(2)}=\sum_{a} \epsilon\left(a_{(2)}\right) a_{(1)},
$$

здесь $\Delta(a)=\sum_{a} a_{(1)} \otimes a_{(2)}$.

Пусть $(A, \Delta)$ - коалгебра. Рассмотрим дуальное пространство $A^{*}$. Пусть $\Delta^{*}:(A \otimes$ $A)^{*} \mapsto A^{*}-$ сопряженное отображение для коумножения $\Delta$. Тогда на пространстве $A^{*}$ можно задать операцию умножения, положив $f g=\Delta^{*} \rho(f \otimes g)$, где $f, g$ - функционалы из $A^{*}$. Пространство $A^{*}$ с заданным умножением называется дуальной алгеброй коалгебры $(A, \Delta)$. Легко видеть, что для любых $f, g$ из $A^{*}$ и любого $a$ из $A$ справедливо равенство

$$
(f g)(a)=\rho(f \otimes g)(\Delta(a))=\sum_{a} f\left(a_{(1)}\right) g\left(a_{(2)}\right) .
$$

Коединица $\epsilon$ коалгебры $(A, \Delta)$ является единицей для дуальной алгебры $A^{*}$.

Дуальная алгебра $A^{*}$ коалгебры $(A, \Delta)$ задает бимодульное действие на пространстве $A$, которое определяется следующим образом:

$$
f \rightarrow a=\sum_{a} a_{(1)} f\left(a_{(2)}\right) \quad \text { и } \quad a<f=\sum_{a} f\left(a_{(1)}\right) a_{(2)},
$$

где $f \in A^{*}$ и $a \in A$.

Нетрудно видеть, что для любых $f, g \in A^{*}, a \in A$ имеют место равенства

$$
f g(a)=f(g \rightarrow a)=g(a<f) .
$$

ПреДЛОЖЕНИЕ 1. Пусть $(A, \Delta)$ - произволъная коалгебра и а-элемент из $А$. Положим

$$
\operatorname{Ann}_{l}(a)=\left\{f \in A^{*} \mid f \rightarrow a=0\right\} \quad u \quad \operatorname{Ann}_{r}(a)=\left\{f \in A^{*} \mid a<f=0\right\} .
$$

Тогда $\operatorname{Ann}_{l}(a), \operatorname{Ann}_{r}(a)$ - замкнутые в конечной топологии подпространства алгебрьь $A^{*}$.

ДоказАтеЛьство. Пусть $a \in A$ и $\Delta(a)=\sum_{a} a_{(1)} \otimes a_{(2)}$. Рассмотрим в $A$ линейное подпространство $U$, порожденное всеми элементами $a_{(2)}$. Тогда размерность пространства $U$ конечна и $U^{\perp} \subseteq \operatorname{Ann}_{l}(a)$. Пусть $g$ принадлежит замыканию пространства $\operatorname{Ann}_{l}(a)$. Рассмотрим окрестность $g+U^{\perp}$ элемента $g$. Тогда $\operatorname{Ann}_{l}(a) \cap\left(g+U^{\perp}\right) \neq \varnothing$. Если $f \in \operatorname{Ann}_{l}(a) \cap\left(g+U^{\perp}\right)$, то $f=g+h$, где $h \in U^{\perp}$. Поэтому $g=f-h \in \operatorname{Ann}_{l}(a)$. Следовательно, подпространство $\operatorname{Ann}_{l}(a)$ замкнуто в конечной топологии.

Аналогично доказывается замкнутость в конечной топологии пространства $\operatorname{Ann}_{r}(a)$. 
Пусть $X, Y, Z$ - линейные подпространства коалгебры $(A, \Delta)$. Положим

$$
\wedge(X, Y, Z)=\{a \in A \mid(\Delta \otimes i d) \Delta(a) \in X \otimes A \otimes A+A \otimes Y \otimes A+A \otimes A \otimes Z\} .
$$

\section{Справедливо следующее}

ПреДЛОЖЕНИЕ 2. Пусть $(A, \Delta)$ - произвольная (не обязательно ассочиативная) коалгебра. Тогда для любых линейных подпространств $X, Y, Z$ из $A$ имеет место равенство

$$
\wedge(X, Y, Z)=\left(\left(X^{\perp} Y^{\perp}\right) Z^{\perp}\right)^{\perp} .
$$

ДоказАтельство. Пусть $f \in X^{\perp}, g \in Y^{\perp}, h \in Z^{\perp}$. Тогда $(f g) h \in\left(X^{\perp} Y^{\perp}\right) Z^{\perp}$ и для любого $a \in \wedge(X, Y, Z)$ имеем

$$
\begin{gathered}
(f g) h(a)=\rho(f \otimes g \otimes h)((\Delta \otimes i d) \Delta)(a) \in f(X) g(A) h(A) \\
+f(A) g(Y) h(A)+f(A) g(A) h(Z)=0,
\end{gathered}
$$

где $f(X)=\{f(x) \mid x \in X\}$. Поэтому $a \in\left(\left(X^{\perp} Y^{\perp}\right) Z^{\perp}\right)^{\perp}$, если $a \in \wedge(X, Y, Z)$.

Обратно, пусть $a \in\left(\left(X^{\perp} Y^{\perp}\right) Z^{\perp}\right)^{\perp}$. Тогда

$$
(\Delta \otimes i d) \Delta(a)=\sum_{i=1}^{n} a_{i} \otimes b_{i} \otimes z_{i}+\sum_{j=1}^{m} u_{j} \otimes v_{j} \otimes w_{j},
$$

где $z_{1}, \ldots z_{n}, w_{1}, \ldots, w_{m}$ линейно независимы и все $z_{i} \in Z$, а элементы $w_{i} \notin Z$. Покажем, что

$$
\sum_{j=1}^{m} u_{j} \otimes v_{j} \otimes w_{j} \in X \otimes A \otimes A+A \otimes Y \otimes A .
$$

Если все $v_{j} \in Y$, то все доказано. Пусть $v_{j_{1}} \notin Y$ для некоторого индекса $j_{1} \leqslant m$. Тогда для некоторого элемента $g \in Y^{\perp}$ имеем $g\left(v_{j_{1}}\right) \neq 0$. Определим функционал $h$ на $A$, полагая $h(Z)=0, h\left(w_{j}\right)=0$ для $j \neq j_{1}$ и $h\left(w_{j_{1}}\right)=1$. Для любого $f \in X^{\perp}$ получаем

$$
\begin{aligned}
(f g) h(a) & =\sum_{i=1}^{n} f\left(a_{i}\right) g\left(b_{i}\right) h\left(z_{i}\right)+\sum_{j=1}^{m} f\left(u_{j}\right) g\left(v_{j}\right) h\left(w_{j}\right) \\
& =f\left(u_{j_{1}}\right) g\left(v_{j_{1}}\right) h\left(w_{j_{1}}\right)=f\left(u_{j_{1}}\right) g\left(v_{j_{1}}\right) .
\end{aligned}
$$

Так как $(f g) h \in\left(X^{\perp} Y^{\perp}\right) Z^{\perp}$, то $(f g) h(a)=0$. Следовательно, $f\left(u_{j_{1}}\right) g\left(v_{j_{1}}\right)=0$ и $f\left(u_{j_{1}}\right)=0$. Поэтому $u_{j_{1}} \in X^{\perp \perp}=X$. Отсюда получаем, что $\sum_{j=1}^{m} u_{j} \otimes v_{j} \otimes w_{j} \in$ $X \otimes A \otimes A+A \otimes Y \otimes A$.

Таким образом, $a \in \wedge(X, Y, Z)$.

ОпредЕЛЕНиЕ. Линейное подпространство $B$ коалгебры $(A, \Delta)$ называется nодкоалгеброй, если $\Delta(B) \subseteq B \otimes B$.

Хорошо известно, что $B$ является подкоалгеброй тогда и только тогда, когда пространство $B^{\perp}$ является идеалом в $A^{*}$. Дуальная алгебра $B^{*}$ коалгебры $(B, \Delta)$ изоморфна фактор-алгебре $A^{*} / B^{\perp}$. Нетрудно показать, что $B$ - подкоалгебра в том и только том случае, когда пространство $B$ есть $A^{*}$-подбимодуль. Если $I-$ идеал алгебры $A^{*}$, то подпространство $I^{\perp}-$ подкоалгебра в $A$. 
Коалгебра $(A, \Delta)$ называется простой, если она имеет только две подкоалгебры: нулевую и саму коалгебру $(A, \Delta)$.

Коалгебра $(A, \Delta)$ называется локально конечномерной, если любой элемент коалгебры $(A, \Delta)$ содержится в конечномерной подкоалгебре.

ОПреДЕЛЕНИЕ. Пусть $\mathscr{M}$ - произвольное многообразие алгебр. Тогда пара $(A, \Delta)$ называется $\mathscr{M}$-коалгеброй, если ее дуальная алгебра $A^{*}$ принадлежит многообразию $\mathscr{M}$.

Хорошо известно (см. [2]-[4]), что всякая ассоциативная, альтернативная, йорданова или структуризуемая коалгебра локально конечномерна.

Пусть $(A, \Delta)$ - коалгебра, $X$ - подпространство в $A$. Определим в $A$ последовательность подпространств $\left\{X_{[n]}\right\}$, полагая

$$
X_{[0]}=0, \quad X_{[1]}=X \quad \text { и } \quad X_{[n+1]}=\wedge\left(X_{[n]}, X_{[n]}, X_{[n]}\right) .
$$

Для подпространства $Y$ дуальной алгебры $A^{*}$ положим

$$
Y^{[0]}=A^{*}, \quad Y^{[1]}=Y \quad \text { и } \quad Y^{[n+1]}=\left(Y^{[n]} Y^{[n]}\right) Y^{[n]}=\left\{\sum_{i}\left(x_{i} y_{i}\right) z_{i} \mid x_{i}, y_{i}, z_{i} \in Y^{[n]}\right\} .
$$

Пусть $U$ - подмножество в $A^{*}$. Тогда для $a \in A$ положим $U(a)=\{f(a) \mid f \in U\}$. В следующем предложении мы полагаем $f \cdot a=f \rightarrow a$ и $a \cdot f=a \leftarrow f$.

ПреДЛОЖЕНИЕ 3. Пусть $(A, \Delta)$ - коалгебра с коединицей $\epsilon, A^{*}$ - дуалъная алгебра коалгебры $(A, \Delta), B$ - подкоалгебра в $A$ и $I=B^{\perp}$. Предположим, что куб любого идеала алгебры $A^{*}$ снова является идеалом. Тогда для любого числа $n$ в коалгебре $(A, \Delta)$ имеет место равенство $B_{[n]}=\left(I^{[n]}\right)^{\perp}$ u $B_{[n]}-$ подкоалгебра в $(A, \Delta)$.

ДокАЗАТЕЛЬСтво. Будем доказывать это предложение индукцией по $n$. Для $n=1$ все доказано. Предположим, что предложение верно для всех $k \leqslant n$. Докажем его для $n+1$. По определению $B_{[n+1]}=\wedge\left(B_{[n]}, B_{[n]}, B_{[n]}\right)$. В силу предложения 2 имеем

$$
B_{[n+1]}=\left(\left(B_{[n]}^{\perp} B_{[n]}^{\perp}\right) B_{[n]}^{\perp}\right)^{\perp} .
$$

По индукционному предположению получаем, что

$$
B_{[n+1]}=\left(\left(\left(I^{[n]}\right)^{\perp \perp}\left(I^{[n]}\right)^{\perp \perp}\right)\left(I^{[n]}\right)^{\perp \perp}\right)^{\perp} .
$$

Для подпространства $X$ из $A^{*}$ через $\bar{X}$ обозначим замыкание $X$ в конечной топологии, т.е. $\bar{X}=X^{\perp \perp}$. Поскольку $B$ - подкоалгебра и куб идеала алгебры $A^{*}-$ снова идеал, то $I^{[n]}-$ идеал в $A^{*}$ для любого числа $n$. Тогда пространство $\overline{I^{[n]}}-$ идеал алгебры $A^{*}$.

Покажем, что

$$
\left(I^{[n+1]}\right)^{\perp}=\left(\left(\left(\overline{I^{[n]}}\right)\left(\overline{I^{[n]}}\right)\right) \overline{I^{[n]}}\right)^{\perp} .
$$

Поскольку $I^{[n]} \subseteq \overline{I^{[n]}}$, то

$$
I^{[n+1]}=\left(I^{[n]} I^{[n]}\right) I^{[n]} \subseteq\left(\left(\overline{I^{[n]}}\right)\left(\overline{I^{[n]}}\right)\right) \overline{I^{[n]}} .
$$

Отсюда получаем, что

$$
\left(\left(\left(\overline{I^{[n]}}\right)\left(\overline{I^{[n]}}\right)\right) \overline{I^{[n]}}\right)^{\perp} \subseteq\left(I^{[n+1]}\right)^{\perp} .
$$


Пусть $a \in\left(I^{[n+1]}\right)^{\perp}$. Пространство $I^{[n]}$ является идеалом алгебры $A^{*}$. Поэтому $\left(\left(A^{*} I^{[n]}\right) I^{[n]}\right) I^{[n]} \subseteq I^{[n+1]}$ и $a \in\left(\left(\left(A^{*} I^{[n]}\right) I^{[n]}\right) I^{[n]}\right)^{\perp}$. Следовательно,

$$
\left.A^{*}\left(I^{[n]} \cdot\left(I^{[n]} \cdot\left(I^{[n]} \cdot a\right)\right)\right)\right)=\left(\left(\left(A^{*} I^{[n]}\right) I^{[n]}\right) I^{[n]}\right)(a)=0 .
$$

Поэтому $I^{[n]} \cdot\left(I^{[n]} \cdot\left(I^{[n]} \cdot a\right)\right)=0$. Отсюда получаем, что

$$
I^{[n]} \subseteq \bigcap_{x \in I^{[n]} \cdot\left(I^{[n]} \cdot a\right)} \operatorname{Ann}_{l}(x) .
$$

По предложению $1 \operatorname{Ann}_{l}(x)$ - замкнутое в конечной топологии подпространство алгебры $A^{*}$. Следовательно,

$$
\overline{I^{[n]}} \subseteq \bigcap_{x \in I^{[n]} \cdot\left(I^{[n]} \cdot a\right)} \operatorname{Ann}_{l}(x) .
$$

Тогда $\overline{I^{[n]}} \cdot\left(I^{[n]} \cdot\left(I^{[n]} \cdot a\right)\right)=0$. Поэтому $\epsilon\left(\overline{I^{[n]}} \cdot\left(I^{[n]} \cdot\left(I^{[n]} \cdot a\right)\right)\right)=0$. Отсюда следует, что $\left(\left(\overline{I^{[n]}} I^{[n]}\right) I^{[n]}\right)(a)=0$ и $\left(\left(\overline{I^{[n]}}\left(A^{*} I^{[n]}\right)\right) I^{[n]}\right)(a)=0$. Следовательно,

$$
A^{*}\left(I^{[n]} \cdot\left(\left(I^{[n]} \cdot a\right) \cdot \overline{I^{[n]}}\right)\right)=\left(\left(\overline{I^{[n]}}\left(A^{*} I^{[n]}\right)\right) I^{[n]}\right)(a)=0 .
$$

Поэтому $I^{[n]} \cdot\left(\left(I^{[n]} \cdot a\right) \cdot \overline{I^{[n]}}\right)=0$ и $\overline{I^{[n]}} \cdot\left(\left(I^{[n]} \cdot a\right) \cdot \overline{I^{[n]}}\right)=0$. Тогда

$$
\left(\left(\left(\overline{I^{[n]}}\right)\left(\overline{I^{[n]}}\right)\right) I^{[n]}\right)(a)=\epsilon\left(\overline{I^{[n]}} \cdot\left(\left(I^{[n]} \cdot a\right) \cdot \overline{I^{[n]}}\right)\right)=0 .
$$

Аналогично получаем, что $\left(\left(\left(\overline{I^{[n]}}\right)\left(\overline{I^{[n]}}\right)\right) \overline{I^{[n]}}\right)(a)=0$. Поэтому $a \in\left(\left(\left(\overline{I^{[n]}}\right)\left(\overline{I^{[n]}}\right)\right) \overline{I^{[n]}}\right)^{\perp}$. Следовательно, $\left(I^{[n+1]}\right)^{\perp} \subseteq\left(\left(\left(\overline{I^{[n]}}\right)\left(\overline{I^{[n]}}\right)\right) \overline{I^{[n]}}\right)^{\perp}$. Отсюда получаем,

$$
\left(I^{[n+1]}\right)^{\perp}=\left(\left(\left(\overline{I^{[n]}}\right)\left(\overline{I^{[n]}}\right)\right) \overline{I^{[n]}}\right)^{\perp} .
$$

Таким образом,

$$
B_{[n+1]}=\left(I^{[n+1]}\right)^{\perp} .
$$

2. Корадикал йордановой (альтернативной) коалгебры. Пусть $(A, \Delta)-$ йорданова (альтернативная) коалгебра и $B$ - простая подкоалгебра коалгебры $(A, \Delta)$. Поскольку коалгебра $(A, \Delta)$ локально конечномерна, то $B$ - конечномерная подкоалгебра. Обозначим через $\operatorname{Corad}(A)$ сумму всех простых подкоалгебр из $(A, \Delta)$. Тогда справедлива следующая

Tеорема. Пусть $(A, \Delta)$ - йорданова (альтернативная) коалгебра с коединицей и $A^{*}$ - дуальная алгебра коалгебры $(A, \Delta)$. Пусть $\operatorname{Rad}\left(A^{*}\right)$ - квазирегулярный радикал алгебры $A^{*}$. Тогда

$$
\operatorname{Corad}(A)^{\perp}=\operatorname{Rad}\left(A^{*}\right) .
$$

Мы докажем данную теорему для случая йордановых коалгебр. Для альтернативных коалгебр доказательство аналогично.

Напомним определение обратимого элемента йордановой алгебры. Элемент $x$ йордановой алгебры обратим с обратным $y$, если $x y=1$ и $x^{2} y=x$.

Сначала докажем несколько вспомогательных лемм. 
Лемма 1. Пусть $(A, \Delta)$ - иорданова коалгебра с коединицей $\epsilon u\left\{B_{n}\right\}-$ возрастающая последовательность подкоалгебр в $A$. Тогда мъ имеем последовательность таких эпиморфизмов $\pi_{n}: A^{*} \mapsto B_{n}^{*}$, что $\operatorname{Ker} \pi_{n}=B_{n}^{\perp}$. Предположим, что $A=\bigcup_{n}^{\infty} B_{n}$. Тогда элемент $f \in A^{*}$ обратим в $A^{*}$ в том и только том случае, когда для любого $n$ элемент $\pi_{n}(f)$ обратим в алгебре $B_{n}^{*}$.

ДокАзАтЕЛЬСтво. Пусть $f \in A^{*}$. Если элемент $f$ обратим в $A^{*}$, то ясно, что для любого $n$ элемент $\pi_{n}(f)$ обратим в алгебре $B_{n}^{*}$.

Предположим, что для любого $n$ элемент $\pi_{n}(f)$ обратим в алгебре $B_{n}^{*}$. Обозначим через $\bar{g}_{n}$ его обратный элемент в алгебре $B_{n}^{*}$. Пусть элемент $g_{n} \in A^{*}$ является прообразом элемента $\bar{g}_{n}$, т.е. $\pi_{n}\left(g_{n}\right)=\bar{g}_{n}$. Рассмотрим эпиморфизм $\phi: B_{n+1}^{*} \mapsto B_{n}^{*}$, полагая $\phi\left(\pi_{n+1}(h)\right)=\pi_{n}(h)$ для любого $h \in A^{*}$. Тогда мы имеем

$$
\pi_{n}(f) \pi_{n}\left(g_{n+1}\right)=\pi_{n}\left(f g_{n+1}\right)=\phi\left(\pi_{n+1}\left(f g_{n+1}\right)\right)=\phi\left(\pi_{n+1}(f) \pi_{n+1}\left(g_{n+1}\right)\right)=1 .
$$

Аналогично $\pi_{n}(f)^{2} \pi_{n}\left(g_{n+1}\right)=\pi_{n}(f)$. Отсюда получаем, что $g_{n}-g_{n+1} \in B_{n}^{\perp}$. Следовательно, $g_{n}(b)=g_{n+1}(b)$ для любого $b \in B_{n}$. Поэтому для $k<l$ мы имеем $g_{k}(b)=g_{l}(b)$ для любого $b \in B_{k}$.

Определим отображение $g: A \mapsto \Phi$, полагая $g(a)=g_{n}(a)$, если $a \in B_{n}$. Предположим, что $a \in B_{k} \cap B_{l}$ и $k<l$. Тогда $g_{k}(a)=g_{l}(a)$. Поэтому отображение $g$ определено корректно. Ясно, что отображение $g$ линейно. Поэтому $g \in A^{*}$. В силу определения функционала $g$ имеем $\pi_{n}(g)=\pi_{n}\left(g_{n}\right)$. Отсюда получаем, что $f g-\epsilon, f^{2} g-f \in B_{n}^{\perp}$ для любого числа $n$. Поскольку $\bigcap_{n}^{\infty} B_{n}^{\perp}=0$, то $f g=\epsilon$ и $f^{2} g=f$. Следовательно, $f$ обратим в алгебре $A^{*}$.

Лемма 2. Пусть $(A, \Delta)$ - йорданова коалгебра с коединицей $\epsilon, B$ - подкоалгебра в $A$ и $I=B^{\perp}$. Предположим, что $A=\bigcup_{n}^{\infty} B_{[n]}$. Тогда $I$ - квазирегулярный идеал алгебры $A^{*}$.

ДокАЗАтЕЛьство. Поскольку $A^{*}$ - йорданова алгебра, то куб идеала алгебры $A^{*}$ - снова идеал. В силу предложения 3 имеем $\left(I^{[n]}\right)^{\perp}=B_{[n]}$. Поэтому $B_{[n]}$ - подкоалгебра коалгебры $(A, \Delta)$ и $B_{[n]}^{\perp}=\left(I^{[n]}\right)^{\perp \perp}$. Следовательно, $\pi_{n}: A^{*} \mapsto B_{[n]}^{*}$ эпиморфизм алгебр, причем Ker $\pi_{n}=B_{[n]}^{\perp}$. Ясно, что последовательность подкоалгебр $\left\{B_{[n]}\right\}$ возрастает. Пусть $f$ - элемент из $I$. Тогда для любого числа $n$ существует такое число $k$, что $f^{k} \in I^{[n]} \subseteq\left(I^{[n]}\right)^{\perp \perp}=B_{[n]}^{\perp}$. Поэтому $\pi_{n}(f)$ - нильпотентный элемент алгебры $B_{[n]}^{*}$. Следовательно, для любого числа $n$ элемент $\pi_{n}(\epsilon)-\pi_{n}(f)$ обратим в $B_{[n]}^{*}$ (см. гл. $\left.14[5]\right)$. Тогда по лемме 1 элемент $\epsilon-f$ обратим в алгебре $A^{*}$.

Таким образом, $I$ - квазирегулярный идеал алгебры $A^{*}$.

Лемма 3. Пусть $(A, \Delta)$ - йорданова коалгебра с коединицей. Тогда $\operatorname{Rad}\left(A^{*}\right) \subseteq$ $\operatorname{Corad}(A)^{\perp}$. Если $B-$ конечномерная подкоалгебра в $A, \operatorname{mo} B \subseteq \operatorname{Corad}(A)_{[n]} \partial л я$ некоторого числа $n$.

ДокАзАтЕЛЬСтво. Пусть $C$ - простая подкоалгебра в $(A, \Delta)$. Поскольку коалгебра $(A, \Delta)$ - локально конечномерна, то $C$ - конечномерная подкоалгебра. Факторалгебра $A^{*} / C^{\perp}$ изоморфна алгебре $C^{*}$, которая является простой конечномерной алгеброй. Поэтому $\operatorname{Rad}\left(A^{*}\right) \subseteq C^{\perp}$. Следовательно, $R\left(A^{*}\right) \subseteq \operatorname{Corad}(A)^{\perp}$.

Рассмотрим коалгебру $(B, \Delta)$. Ее дуальная алгебра $B^{*}$ конечномерна. Поэтомy $\operatorname{Rad}\left(B^{*}\right)$ - пересечение максимальных идеалов алгебры $B^{*}$. Отсюда получаем, 
что $\operatorname{Rad}\left(B^{*}\right)=\operatorname{Corad}(B)^{\perp} \cdot \operatorname{Taк~как~} \operatorname{Rad}\left(B^{*}\right)^{[n]}=0$ для некоторого числа $n$ (см. гл. $15[5])$, то в силу предложения 3 получаем, что

$$
0=\operatorname{Rad}\left(B^{*}\right)^{[n]}=\left(\operatorname{Corad}(B)_{[n]}\right)^{\perp} .
$$

Следовательно, $B=\operatorname{Corad}(B)_{[n]}$.

Как легко видеть, $\operatorname{Corad}(B) \subseteq \operatorname{Corad}(A)$. Индукцией по числу $n$ получаем, что

$$
\begin{aligned}
\operatorname{Corad}(B)_{[n+1]}= & \wedge\left(\operatorname{Corad}(B)_{[n]}, \operatorname{Corad}(B)_{[n]}, \operatorname{Corad}(B)_{[n]}\right) \\
= & ((\Delta \otimes i d) \Delta)^{-1}\left(\operatorname{Corad}(B)_{[n]} \otimes B \otimes B\right. \\
& \left.\quad+B \otimes \operatorname{Corad}(B)_{[n]} \otimes B+B \otimes B \otimes \operatorname{Corad}(B)_{[n]}\right) \\
\subseteq & ((\Delta \otimes i d) \Delta)^{-1}\left(\operatorname{Corad}(A)_{[n]} \otimes A \otimes A\right. \\
& \left.\quad+A \otimes \operatorname{Corad}(A)_{[n]} \otimes A+A \otimes A \otimes \operatorname{Corad}(A)_{[n]}\right) \\
= & \operatorname{Corad}(A)_{[n+1]} .
\end{aligned}
$$

Следовательно, $B \subseteq \operatorname{Corad}(A)_{[n]}$ для некоторого числа $n$.

ДоКАЗАТЕЛЬСТво тЕОРЕМЫ. В силу леммы 3 достаточно доказать, что $\operatorname{Corad}(A)^{\perp}-$ квазирегулярный идеал алгебры $A^{*}$. Для любого числа $n$ положим $B_{[n]}=\operatorname{Corad}(A)_{[n]}$. В силу предложения $3\left\{B_{[n]}\right\}$ - возрастающая последовательность подкоалгебр в $A$. По лемме 3 любая конечномерная подкоалгебра из $A$ содержится в $\operatorname{Corad}(A)_{[n]}$ для некоторого $n$. Поэтому $A=\bigcup_{n}^{\infty} B_{[n]}$. Применяя лемму 2 , получаем, что $\operatorname{Corad}(A)^{\perp} \subseteq \operatorname{Rad}\left(A^{*}\right)$. По лемме $3 \operatorname{Rad}\left(A^{*}\right) \subseteq \operatorname{Corad}(A)^{\perp}$.

Таким образом, $\operatorname{Corad}(A)^{\perp}=\operatorname{Rad}\left(A^{*}\right)$.

\section{СПИСОК ЦИТИРОВАННОЙ ЛИТЕРАТУРЫ}

[1] H. G. Heyneman, D. E. Radford, "Reflexivity and coalgebras of finite type", J. Algebra, 28 (1974), 215-246.

[2] J.A. Anquela, T. Cortes, F. Montaner, "Nonassociative coalgebras", Comm. Algebra, 22:12 (1994), 4693-4716.

[3] M. E. Sweedler, Hopf Algebras, Math. Lecture Note Series, W. A. Benjamin Inc., New York, 1969.

[4] В.Н. Желябин, "Структуризуемые коалгебры”, Алгебра и логика, 35:5 (1996), 529542.

[5] К.А. Жевлаков, А. М. Слинько, И. П. Шестаков, А. И. Ширшов, Колъца, близкие к ассоциативным, Современная алгебра, Наука, М., 1978.

\section{В. Н. Желябин}

Институт математики СО РАН

E-mail: vicnic@math.nsc.ru
Поступило

07.07.2005

Исправленный вариант

08.12 .2005 